Journal of Theoretical and Applied Sciences
(ISSN:2637-692X)

\title{
Gravity defined precisely with quarks - squaring the circle to achieve fine description of black hole physics, and understanding the formation of key constellations
}

Florent Pirot

Independent researcher

\begin{abstract}
Gravity is redefined by drawing on the author's works and inputting quark physics in a way consistent with it ; it allows to better understand black hole dynamics, black hole eruptions and some constellation patterns in the sky (the Scorpio, Ursa Minor and Ursa Major as well as Cassiopea for instance). The concept of gravitational lensing is rapidly differenciated from black hole eruptions. Some final remarks underline a possible threat for missions to the Moon.
\end{abstract}

Keywords: black hole physics, gravity, antigraviton, particle physics, constellations, cosmology
*Correspondence to Author:

Florent Pirot

Independent researcher

How to cite this article:

Florent Pirot. Gravity defined precisely with quarks-squaring the circle to achieve fine description of black hole physics, and understanding the formation of key constellations. Journal of Theoretical and Applied Sciences, 2021, 4:21.

\section{eScîPub}

eSciPub LLC, Houston, TX USA.

Website: http://escipub.com/ 
The key initial postulate is the negative rest mass of the down quark. This relies on a long personal experience of neutron physics. In other words, at rest the down quark moves. If it is on a weighter, it creates a thin flux that succions the weighter up. The neutron itself carrying two down quarks and one up quark also has a slightly negative rest mass (unlike the proton). To sum up, the proton is subject to the laws of gravity, but the neutron "flies" away in a way tampered by its carrying of one up quark (so a spin of $2 / 3$ will apply in general to its flight). This also means that with a powerful enough thermonuclear test on Earth, a number of neutrons will escape its gravity.

Down quarks have an antiparticle (the down antiquark, or antidown) and this antiparticle is the key of black hole physics. This antiparticle is the antigraviton itself. With the isospin of $1 / 2$ of the down quark, an anti-spin has to be inprinted to comprehend the antidown. The spin rolls back onto itself and creates a S-shape particle because symetrically, the other side of the particle is also pulled in.

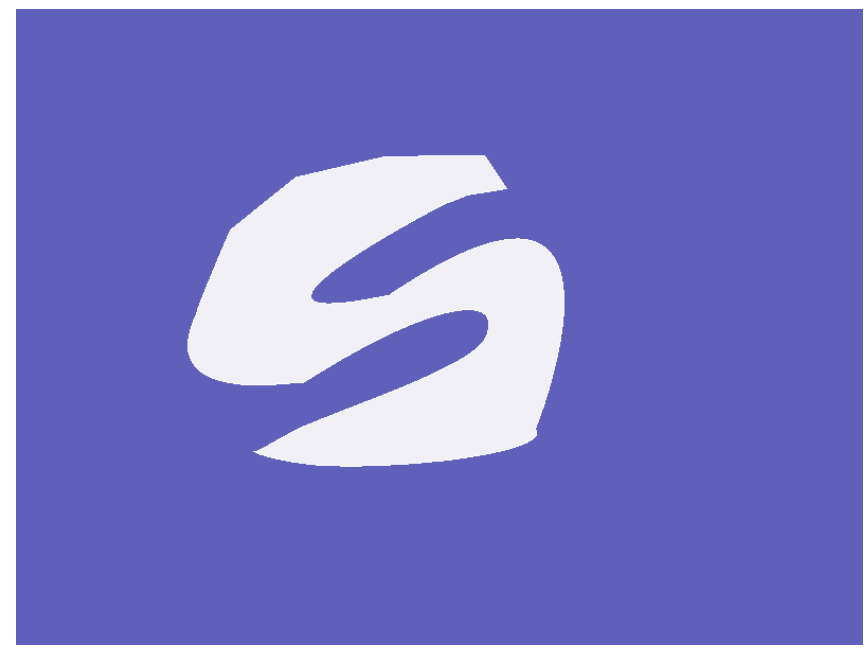

Illustration 1: Fig 1. The antidown, or antigraviton, in white

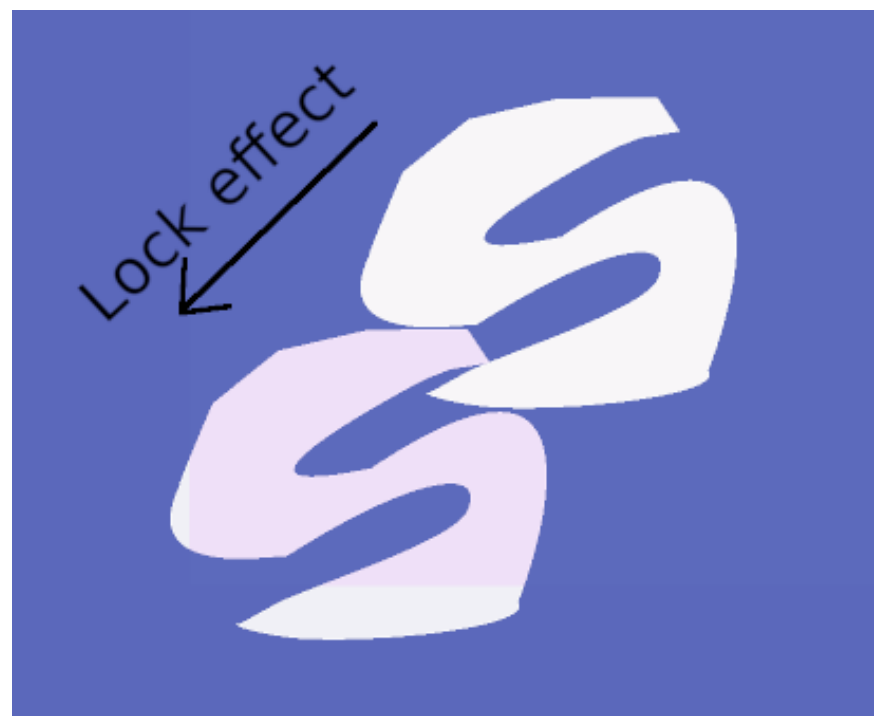

Illustration 2: Fig 2. Series of antidowns (antigravitons) hooking up to each other, they will keep the same properties (two openings for each cluster). 
The antidown, or antigraviton is in permanent spin onto itself, always ever closing with the center spinning faster and inertia on the "swabs ». It creates a " hook » effect. This hook can catch other antidowns (antigravitons are « attracted to each other »[1]), or up quarks. Indeed the natural attraction between down and up quarks is kept and the antidown (antigraviton) attracts the up.

An antigraviton, or series of, always keeps the ability to catch two up quarks, conforming with the structure of the proton (two up, one down quark). An homothetical parallel of the proton is stabilized through this way in the black hole : there is no brutal change, inversion keeps atomic properties. Neutrons however are not as easy to absorb in as they fly and are not naturally brought into black holes. Neutrons have no affinity for antidowns as they are already dominated by down quarks and, whereas antidowns are attracted to each other down quarks are not attracted to antidowns. They tend to bounce back when they impact antidowns. Hook-up is difficult to quasi impossible except under very strong pressures and may not hold anyway. This explains why in black holes neutrons travel slowly, in " meta-mobility ", they represent the motor of the black hole dynamism, and cause of black hole eruption when they slowly fission superheavy atoms formed through the high pressure merger within in a sustained way. Black hole eruptions were described in [1] and more precisely in [2] already. Neutrons can (and will) also nevertheless frequently convert into their antiquarks within a black hole. The down particle travelling in the middle of antigraviton chains has a spiralling shape with conical ways (open angles, as an elongated $\mathrm{W}$ ) and any impact of a third particle onto such a down quark will be enough, in this densely compressed environment, to give it the supplementary spin needed to turn it into its antiparticle, quickly chained with the other antigravitons.

This definition of the antigraviton also allows to comprehend why some tenuous gravity changes led to the idea, developed by Los Alamos scientists such as Edward Teller, that temporary black holes appeared during atomic tests in the precise locus of fission under implosion. It is indeed certain that down quarks will be temporarily disrupted, their spin partially inverted, forming small chains of pseudoantigravitons in a nonsustained way, but the quasi immediate dissipation of energy leads to decompression and absence of bonding.

The high inertia of the swabs, relative to the internal rotation of the central part of the antidown (akin to the internal spin of the free neutron in squeezed state actinides (fissile atoms) [3]) is a crucial element in its selfsustainability. This explains why the antigraviton-to-antigraviton link cannot be torn apart easily. In fact, only a neutron impact can break a chain (as for atoms : only neutrons can fission heavyweight atoms, and it was underlined the homothetical parallel kept within the black hole for atomic properties), and a slow one only (whereas the inertia wheel of the central spin allows antigravitons to absorb rapid neutron impacts, a slow neutron impact pressing on the lock point is likely to be able to 'un-lock' the two antigravitons, which will then absorb energy as the release of the swabs decompensates the passivity of the bond associated to antidown dilatation into each other - the « arms » come back, the energy is brought back into the central part, rotating it faster). The antigraviton chains mixed with each other can be seen as a kind of spaghetti bowl where the pasta is twirled more and more. When they are strongly interlocked, the internal spin in each antigraviton is strongly limited by that interlocking but energy is transferred to the 
whole group, in a joint movement of rotation that is (when this group is the entire black hole) the black hole's rotation. When a chain is broken, it frees up the central wheel of each liberated antigraviton.

Pions in the black hole certainly also contribute to the binding in the way they contribute to the strong nuclear force outside, giving to the whole its stability in spite of the variations in rotation.

\section{Fermionic condensates of neutrons impacting black hole : the emergence of constellations such as the Scorpio, Ursa Minor and Ursa Major}

This observation allows to confirm the initial postulate and to square the circle. Fermionic condensates of relativistic neutrons were described, in geophysics, in [4]. Such Fermionic condensates of relativistic neutrons can also form as they travelling through radioactive matter before impacting a black hole, by cruising in the high atmosphere of a star for instance or through an intense supervolcanic cloud on a planetary object. A few neutrons in a Fermionic condensate then entering a black hole have a low but existing probability of triggering its eruption, in a pattern that will be quasi identical to the geophysical pattern described in [4]. The neutron "beam » descends in the black hole, impacts objects (groups of superheavy atoms formed by the mergers, bound with their antigravitons, expelled to form the primitive moons, planets or stars, in the way described in [1]) that have a progressively denser nature (as the beam reaches a more central point in the black hole), before being pushed out while decondensating as the Fermionic bond has dissipated - the slower neutrons scatter, kicking out less dense objects in the last part of their travel, before going out as well or more likely staying in the black hole. Unlike in typical black hole eruptions where a crown of stars shape is achieved around it, the sinuous pattern is translated in the organization of the erupted stars. The Scorpio constellation is a particularly significant example of the phenomena. Ursa Major and Ursa Minor are also products of such an event (with time having passed and stars having moved somehow) and other constellations also indicate such an event.

\section{Cassiopea}

The " elongated $\mathrm{W}$ " shape of the down particle pressed inbetween antigravitons indicates the obvious origin of Cassiopea : such a down quark having been kicked out by internal spin of the antigraviton chains. It is obviously a local unlocking of a small group of antigravitons liberating the elongated down quark that was spiralling around - it returns to its non-elongated nature brutally and then resonates, taking back for some time most of its elongated shape, going then back and forth in less and less intense ways until stabilization ; its kicks have transferred through quantum mechanics the elongated $W$ shape away to other antigraviton chains and their associated objects leading to a black hole eruption event of the same shape ; the initial event has transferred a movement reproduced (in an exponential way) by the " pyramid » of heavy atoms (associated with their pressuring antigravitons) above it. The constellation of Cassiopea is opposite the panhandle of Ursa Minor, indicating they have the same black hole as origin. This W-shape eruption can only happen in an unperfectly stabilized black hole where several antigraviton chains have not locked into each other (as opposed to the unity with a single extraordinary long chain of antigravitons after long term rotation forming a very consistent black hole), and this is also consistent with the same black hole having underwent another eruption beforehand (which is why Cassiopea is indeed facing 
Ursa Minor, and they have a single black hole as common origin).

\section{Draco, the Hydra, Pisces, Cetus, Aquarius, Sagittarius...}

These constellations also presents the clear signs of a Fermionic condensate of relativistic neutrons impacting a black hole, as for Ursa Major, Ursa Minor and the Scorpio. But the elongation is so important that the factor of a random association of stars remains another causal possibility.

These prolegomenas allow general deductions for many other constellations that present a mix of these causal patterns as joint origin.

\section{Gravitational lenses ("Einstein Rings ») or black hole eruptions ?}

The phenomena of gravitational distorsion of the visual field, by a black hole in front of a group of stars, creating a distorsion called gravitational lensing (a phenomena predicted by Albert Einstein) has to be dissociated from the black hole eruptions of a crown shape that may present semisimilar aesthetics. This is for instance exemplified in a recent ESA / Hubble (NASA) picture ${ }^{1}$ that actually is a black hole eruption, quite identical to the example studied in [2] ; there is absolutely no deformation of the galaxies around (unlike for instance in the case of GAL-CLUS-02205-38303, an actual Einstein Ring).

\section{One last comment :}

It is certain that a small group of antigravitons is aggregated between the Moon and the Earth. A number of observations have shown tenuous losses of luminosity that it would be absurd to link to

\footnotetext{
${ }^{1}$ Nasa.gov/sites/default/files/thumbnails/image/Potw 1814a.jpg

${ }^{2}$ Retina variations while looking precisely at these blackening areas were also noted, attraction in that direction of the superthin corneal tissue was
}

airborne alpha-emitting nanoparticulates ${ }^{2}$. A microscopic black hole is the explanation. It appears indeed as a kind of "vortex", the word used in [5] to describe "reasons for removal of the Moon "; whereas the argument on a removal of the Moon from antigravitons lodged inbetween Earth and the Moon is absurd, there clearly is a small antigraviton vortex and it is entirely consistent with the physics of antigravitons and their attraction by other antigravitons (those of the core of the Earth and those of the core of the Moon).

The installation of a linear particle accelerator on the Moon could be an idea to better observe the evolutions of this tiny black hole over time (by firing it to observe variations in particle path) but that tiny black hole also has to be understood as representing somehow of a threat for missions on the Moon. And also certainly as origin for many cosmic fireballs.

\section{References :}

[1]. Pirot F, "Nucleosynthesis and star \& planet formation in black holes, explosive and effusive volcanism, geochemistry, bolides, the graviton / antigraviton couple, and spontaneous explosion of nuclear reactors", in From an Einstein Syndrome to the People, Editions universitaires européennes, 2019.

[2]. Pirot F, A Rule of Natural Disasters Coming to Rebalance Unjust Development and Irresponsibility-Laws of Physics Explain the Trope of "Divine Retribution" against the "Nouveaux Riches", International Journal of Physics. 2019, 7(4), 135-140. doi: 10.12691/ijp-7-4-4

[3]. Pirot F, Fissile vs. Fertile Atoms Renamed Squeezed State vs. Anti-squeezed States, Why Uranium, Thorium Etc are Invisible in Star Spectrometry Data, and a Return on

observed and it is clear that the high density of keratoconus cases that is well known in Arabic populations is related to moon observation customs. 
Human Behaviour, Cultural Insecurity Defined as Anti-squeezed Behaviour, Linked to Brain Trauma, International Journal of Physics. 2020, 8(3), 108-113. DOI: 10.12691/ijp-8-3-4

[4]. Pirot F, Fermionic Condensation Explains the Formation of Subcontinents and Small
Volcanic Islands around Them - General Geophysical Rules. International Journal of Physics. 2021; 9(1):42-47. doi: 10.12691/ijp9-1-5

5. S. Orlov. REASONS FOR REMOVAL OF THE MOON. International Research Journal of Physics, 2018, 1:4 